\title{
Werteorientierte Effizienz und Unternehmensführung Stärkung von Stationsteams
}

Die Leistungsverdichtung in der Psychiatrie wird von Mitarbeitenden zunehmend thematisiert. Doch das Ende der Fahnenstange ist noch nicht in Sicht. Ein leistungsorientiertes pauschalierendes Vergütungssystem auf der Grundlage von tagesbezogenen Entgelten oder anderen Abrechnungseinheiten soll ab 2013/2014 zunächst in freiwilligen Optionsjahren budgetneutral umgesetzt werden. Ab 2015 wird der Beginn der Konvergenzphase sein, 2017 erfolgt der endgültige Startschuss - die verpflichtende Umsetzung des neuen Vergütungssystems.

\section{Steigender Effizienzdruck}

Heute ist vieles noch unkonkret. Klar ist nur, dass auf die Einrichtungen neue Anforderungen an Dokumentation, Organisation und Wirtschaftlichkeit zukommen. Gerade unzureichend koordinierte Abläufe rund um die Patientenversorgung zwischen dem betreuenden multiprofessionellen Team kosten Zeit, schaffen unnötige Belastungsspitzen und sorgen künftig gegebenenfalls sogar für Erlöseinbußen. Diese Entwicklung macht auch vor Privatkliniken nicht halt. Im Jahr 2010 führte die Pflegedienstleitung der Privatklinik Dr. Amelung eine gezielte Befragung des Pflegepersonals durch. Die Auswertung dokumentierte folgende Anliegen zur Verbesserung der Rahmenbedingungen:

- Reduzierung des administrativen Aufwands und der patientenfernen Aufgaben

- Erhöhung der Kontaktzeiten mit den Patienten

- Verbesserung der Kommunikation und des patientenbezogenen Informationsflusses zwischen den Berufsgruppen

- Stärkung der Teamidentifikation über die Berufsgruppen hinweg

Die kontinuierliche Patientenbefragung weist nach wie vor eine sehr hohe Patientenzufriedenheit aus. Trotzdem nahm die Klinikleitung die Belastungsklagen der Mitarbeitenden im Pflegedienst auf Station und ihre lösungsorientiert formulierten Anliegen als Anlass, das Organisationskonzept der Klinik auf den Prüfstand zu stellen.

\section{Individuelle Patientenbehandlung} wird großgeschrieben

In der Privatklinik Dr. Amelung werden Patienten seit über 100 Jahren medizi- nisch betreut. Das Wohlergehen und die Zufriedenheit des Patienten sind Maßstab und oberstes Ziel der Privatklinik. Diagnostik, Therapie und ebenso die tägliche Betreuung sind individuell auf jeden einzelnen Patienten ausgerichtet. Die seit Generationen gereifte Erfahrung im Umgang mit ihren Patienten, das exklusive Ambiente und die persönliche Art des Miteinanders bieten den Rahmen für eine hoch professionelle Behandlung. Die familiäre Atmosphäre auf Station bedeutet den meisten Patienten genauso viel wie das hochqualifizierte individuelle Behandlungsangebot. So wird jeder Patient stets von der Leitenden Psychologin selbst gesehen und individuell - orientiert an den jeweiligen ärztlichen Therapieempfehlungen und seinen eigenen Bedürfnissen - von den vielfältig spezialisierten Psychotherapeuten dem jeweils optimal passenden zugeordnet. Die Zuordnung richtet sich nicht nach der Station, auf welcher der Patient untergebracht ist. Vielmehr wird durch eine stimmige Passung hochqualifiziertes Personal erst optimal zur Wirkung gebracht. Gerade diese Kernkompetenz trägt zu einer wirksamen Behandlung bei und führt dazu, dass die Patienten am Ende ihres Aufenthaltes sich sowie ihrer Umwelt neu gestärkt begegnen. Diese Bandbreite an Individualität und die Vielfalt des therapeutischen Angebots sind eine Stärke, welche die Klinik in ihrem Markt abhebt.

\section{Ein Luxus, den sich auch}

\section{Privatkliniken heute kaum noch} leisten können?

Das Angebot ist gefährdet, dem wachsenden Effizienzdruck zum Opfer zu fallen. Indem die Patienten stationsfern von ihren individuell zugewiesenen Ärzten und Therapeuten betreut werden, entsteht 
enormer Koordinationsaufwand. Den Koordinationsaufwand muss die Station welche die temporäre Heimat des Patienten bildet - und damit zum Großteil die Pflegekräfte ausgleichen. Patienten stellen immer höhere Ansprüche an die Betreuungsintensität. Die Aufenthaltsdauern und die verfügbaren Mittel werden gleichzeitig immer geringer. So war die Privatklinik gefordert, nach Lösungen zu suchen, die weiterhin genügend Raum für ein individuell zugeschnittenes Behandlungsangebot bieten. Dabei muss dies erlauben, dass sich hochqualifiziertes Personal auf seine Kerntätigkeit konzentriert und Stationen administrativ entlastet werden. Denn schnell war klar, dass eine Einschränkung des patientenindividuellen Leistungsangebotes für die Privatklinik außer Frage steht. Also war die Devise, der „Verschwendung“ von Arbeitszeit genauer auf die Spur zu kommen und damit den formulierten Anliegen der Mitarbeiter näher zu rücken. In einer besseren organisatorischen Koordination der Berufsgruppen und der verzahnten Zusammenarbeit der Bereiche sollten diese schlummernden Potenziale gehoben werden. Sind z.B. die Pflegenden unnötig einen hohen Anteil ihres Tages telefonisch mit Koordinationsaufgaben gebunden oder damit beschäftigt, Informationen „hinterherzueilen“, dann fehlt diese Zeit für den direkten Kontakt mit dem Patienten. Gerade die Stärken der Mitarbeiter auf Station entfalten sich meist in der direkten Arbeit mit dem Patienten. In den Zeiten massiver Leistungsverdichtung sind sie die größten Schätze einer Klinik.

So entwickelte die Klinikleitung die Vorstellung, die Organisation auf ein Stationsarztsystem umzustellen, indem die behandelnden Ärzte - anders als die Psychologen - fest auf einer Station eingesetzt werden. Da auf eine individuell stimmige Arzt-Patienten-Zuordnung nicht verzichtet werden soll, müssen Patienten so bereits bei der Einbestellung nach Abstimmung mit Einweiser oder nach Kenntnis der Vorgeschichte der am besten geeigneten Station zugewiesen werden. Bei Vollauslastung des Hauses ist dieses Belegungsmanagement keine einfache Aufgabe. Den Ärzten wird es dadurch jedoch möglich, ihren Arbeitstag fest auf ihrer jeweiligen Station zu verbringen, und damit unmittelbarer Ansprechpartner für das Stationsteam zu sein - bzw. wesentlich stärker als in der Vergangenheit selbst Teil des Stationsteams zu werden. Dadurch kann sich der patientenbezogene Informationstausch wesentlich vertiefen und die Organisationsverantwortung für die Station können von Arzt und Pflege unmittelbar gemeinsam und auf Augenhöhe ausgeübt werden.

\section{Projektumsetzung}

In einem von Ruhl Consulting extern begleiteten Pilotprojekt wurde nach dieser Grundsatzentscheidung in mehreren Sitzungen unter Beteiligung beider Leitenden Ärzte und der Pflegedienstleitung ein Konzept ausgearbeitet, welches dezidiert die wesentlichen Anliegen auf Station aufgriff. Gleichzeitig sollte die Chance der Veränderung genutzt werden, bewusst klare Führungsstrukturen und schnelle Entscheidungen und damit die Entwicklung von Stationsteams zu fördern.

Die Umsetzung kann nicht über die Köpfe der Mitarbeiter hinweg erfolgen. Um eine starke Identifikation der Mitarbeiter der unterschiedlichen Berufs- und Interessengruppen mit ihrem jeweiligen Stationsteam zu erreichen, ist der Prozess der Teamentwicklung eine wichtige Phase. Diese braucht vor allem Zeit. Zentraler Erfolgsfaktor ist hierbei eine wertschätzende berufsgruppenübergreifende Zusammenarbeit. Die gegenseitige Ergänzung und das Miteinander auf Augenhöhe fördern die Identifikation und die Zufriedenheit innerhalb der Stationsteams. Im Rahmen des Projektes wurde zusammen mit den Mitarbeitern der verschiedenen Berufsgruppen ein Stationshandbuch erarbeitet - zunächst für die Pilotstation, beim späteren ProjektRollout dann auch für die restlichen Stationen - in welchem Verbindlichkeiten und Regelungen zum strukturierten Tagesablauf auf Station vereinbart wurden. Im Ergebnis des Pilotprojektes zeigte sich, dass eine hohe Disziplin bei den beteiligten Ärzten und Pflegekräften vorhanden war, die gemeinsamen Vereinbarungen einzuhalten. Insbesondere die Einführung der Regelkommunikation im Stationsteam (morgendliche Kurzübergabe, wöchentliche Kurvenvisite) wird sehr wertgeschätzt. Die Ärzte konzentrierten sich auf die geplanten Zeitfenster mit ihren Patienten und konnten so die gewünschte hohe Frequenz von Arzt-Patienten-Kontakte einhalten und teilweise sogar übertreffen. Die Entlassungen der Folgewoche werden zuverlässig vorher besprochen. Auch die wöchentliche Kurvenvisite wird gewissenhaft durchgeführt, um so den patientenbezogenen Informationsaustausch im Stationsteam zu festigen, die Übergänge der Therapiephasen $\mathrm{zu}$ besprechen und die Entlassungen vorzubereiten. Nach Abschluss der Evaluation der Pilotphase wurde der Rollout auf die anderen Stationen gestartet. Die Schwerpunkte in den anschließenden Workshops wurden nach den Themen ausgerichtet, die den jeweiligen Stationsteams am meisten „unter den Nägeln brannten.“ Grundsätzlich war in allen Sitzungen ein hoher Wille aller mitarbeitenden Ärzte und Pflegekräfte zur aktiven Beteiligung am Veränderungsprozess spürbar.

Eine verbindliche Grundstruktur im Tagesablaufschema der Station in der koordinierten Zusammenarbeit der Berufsgruppen und die gegenseitige Organisationsverantwortung verringern den Absprachebedarf und helfen somit täglich Zeit zu sparen. In Psychiatrien mit einer hohen Schlagzahl an Behandlungen, wie in der Privatklinik Dr. Amelung, ist vor allem die Koordination der vielfältigen Behandlungen durch Ärzte und Therapeuten ein wichtiges Thema. Das Stationsarztsystem basiert auf der Idee, dass die Ärzte auf festen Stationen eingesetzt werden, welche wiederum den Leitenden Ärzten der Klinik zugeordnet sind. Diese Zuordnung ermöglicht, klare Führungsstrukturen zu etablieren und schnelle Entscheidungen möglichst nah an der Basis zu treffen ohne den Blick für das Ganze zu verlieren. Innerhalb der jeweiligen Arztgruppe entscheiden die Leitenden Ärzte autonom übergreifende Entscheidungen werden jedoch nach wie vor gemeinsam getroffen. Durch den räumlich konzentrierten Einsatz der Ärzte werden die Laufwege der Ärzte verringert, die Entwicklung von Stationsteams und die Übernahme von Organisationsverantwortung gefördert. Der Stationsarzt ist der zentrale Wissensträger über die Behandlungsprozesse der Patienten auf der Station und trägt Verantwortung für die tägliche Informationsweitergabe an das Pflegeteam seiner Station ebenso wie an die behandelnden Psychologen. Eine zielgerichtete Kommunikation ist zentral für das Management der Station. So ist es gelungen, einen Lösungsansatz zu finden, der nicht davon abhängt, die Therapeuten den Stationen zuzuordnen und dennoch stationsspezifischen Informationsrückfluss zu liefern. Das Behandlungsteam tauscht sich in strukturierten Klinikkonferenzen patientenbezogen aus. Über diesen und verschiedene andere Kommunikationskanäle soll sichergestellt sein, dass die Station über die 
Besonderheiten der jeweiligen Therapeuten informiert ist. Für die Information der Patienten sind Informationsgruppen organisiert.

4 Monate nach dem Rollout zeigt eine erste Evaluation einen extrem hohen Zuspruch der Mitarbeiter aller Berufsgruppen zur neuen Organisationsform. Die Zufriedenheitswerte mit dem Stationsarztsystem sind exzellent - und das in Wochen, die durch hohe Ausfallquoten in der Pflege einerseits und durch hohe Anforderungen der Ärzte an die Individualität der Patientenbehandlung andererseits geprägt sind. Die Evaluation ergab, dass die neu definierte Regelkommunikation (morgendliche Kurzübergaben und Kurvenvisiten) zu $100 \%$ eingehalten wird. Auch in der Mitarbeiterbefragung wird diesem geregelten Informationsaustausch eine große Bedeutung zugesprochen. Durch dieses Instrument wird sichergestellt, dass zentrale Patienteninformationen zwischen Arzt und Pflege übergeben werden.

Allerdings sehen nicht alle Mitarbeiter durch das Stationsarztsystem frei gewordene Kapazitäten, welche unmittelbar der Patientenversorgung zu Gute kommt. Dies ist jedoch nach Aussage der Mitarbeiter eher auf die hohen Ausfälle im Pflegedienst zurückzuführen, als auf das eingeführte Stationsarztsystem. Zudem

\section{Korrrespondenz}

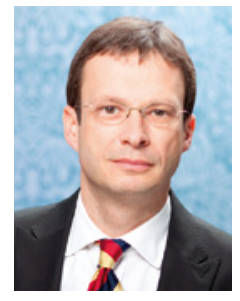

Dr. med. KlausChristopher Amelung

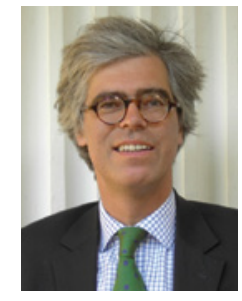

Dr. med.

Florian Nicolai Brandt

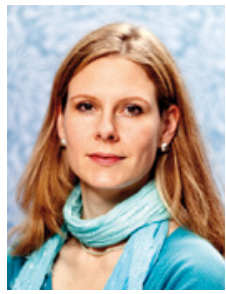

Dipl.-Pflegewirtin (FH) Sandra Ness

konnten im Rahmen des Projekts nicht alle Schnittstellen bearbeitet werden, wodurch es in diesen Bereichen teils zu einem höheren Koordinationsaufwand insbesondere für die Pflege kommt.

Die Mitarbeitenden arbeiten trotz mancher Schwierigkeiten äußerst konstruktiv an der weiteren Verbesserung der berufsgruppenübergreifenden Ablauforganisation und den diesbezüglichen Rahmenstrukturen weiter.

Gelebte Organisationsentwicklung Ein Zusammenwachsen als Team ist auf allen Stationen deutlich wahrnehmbar. In den Teams herrscht eine z. T. sehr harmonische, konstruktive Arbeitsatmosphäre. Durch strukturierte Abläufe zwischen den Berufsgruppen und den Abbau an Schnittstellen konnten Redundanzen vermieden und dadurch Raum in Zeiten hoher Arbeitsverdichtung geschaffen werden. Der Projekterfolg ist auf den Einsatz der Führungskräfte für das Stationsteam und die damit verbundene Würdigung der Mitarbeitenden zurückzuführen. Durch den offenen Dialog zwischen Führungskräften und Mitarbeitenden wird bei allen Höhen und Tiefen - auch eine deutliche Effizienzsteigerung bei weiterhin höchst individueller Patientenbehandlung tatsächlich erlebbar.

Privatklinik Dr. Amelung GmbH

Altkönigsstraße 16

61462 Königstein im Taunus

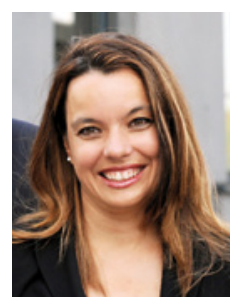

Dr. Elke Eberts,

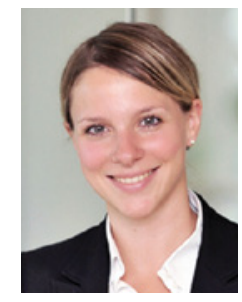

Alexa Schmall

Ruhl Consulting AG

Strategy \& Realisation

Eastsite II

Harrlachweg 1

68163 Mannheim

E-Mail: info@ruhl-consulting.de 